\title{
Error motion of a kinematic spindle
}

\author{
King-Fu Hii ${ }^{a}$, R. Ryan Vallance ${ }^{b, *}$, Robert D. Grejda ${ }^{c}$, Eric R. Marsh ${ }^{c}$ \\ ${ }^{a}$ Precision Systems Laboratory, University of Kentucky, 015 Ralph G. Anderson Building, Lexington, KY 40506, USA \\ ${ }^{\mathrm{b}}$ Precision Systems Laboratory, The George Washington University, 738 Academic Center, 801 22nd St., NW, Washington, DC 20052, USA \\ ' Machine Dynamics Research Laboratory, The Pennsylvania State University, 21 Reber Building, University Park, PA 16802, USA
}

Received 21 January 2003; received in revised form 10 October 2003; accepted 7 November 2003

\begin{abstract}
A prototype spindle for low-force, low-speed applications that is kinematically constrained in accordance with the principles of exact constraint was designed, fabricated, and tested. In the prototype spindle, the position and orientation of the shaft (rotor) are constrained at five contact points; four constraints are arranged radially around the rotor, and one constraint is located at the end of the rotor. This paper describes the design of the prototype spindle and presents a study of its error motion for the hardened steel rotor sliding on brass and UHMW-PE bonded to ceramic. The error motion is observed to be highly repeatable with less than $100 \mathrm{~nm}$ asynchronous errors, and a simple analytical model enables the prediction of the synchronous error motion using measurements of the rotor's roundness profiles.

(C) 2003 Elsevier Inc. All rights reserved.
\end{abstract}

Keywords: Spindle; Kinematic constraint; Exact constraint; Axis of rotation; Error motion

\section{Introduction}

In many high-precision machines and instruments, linear motion is guided by bearings that are arranged in a kinematic or exact-constraint fashion [1]. Such bearing arrangements constrain the motion of a translating body in five degrees of freedom but leave one degree of freedom unconstrained so that the body can translate along a linear trajectory. Blanding [2] provides a systematic method for designing the location and direction of the constraints in precision machines. Kinematic arrangements help assure repeatable and reproducible error motion along the trajectory, and then accurate motion is attainable using bearing components with high form accuracy. Some high-precision instruments have used kinematic arrangements with sliding contact between the translating and stationary bearing elements. For instance, a kinematic sliding-contact linear bearing was used in the Nanosurf 2 [3] profilometer that was later offered commercially by Rank Taylor Hobson as the Nanostep instrument. A similar design is also employed in the linear bearings for the NIST molecular measuring machine [4].

Considering the success of kinematic arrangements of sliding-contact linear bearings, a spindle that uses sliding-contact constraints arranged in a kinematic fashion might be useful for achieving high-precision rotary motion.

\footnotetext{
${ }^{*}$ Corresponding author. Tel.: +1-202-994-9830; fax: +1-202-994-0238.

E-mail address: vallance@gwu.edu (R.R. Vallance).
}

However, such spindles have received little investigation. Therefore, the objective of this work was to assess the feasibility of a kinematically constrained spindle for attaining high-precision rotary motion in low-force and low-speed applications. This objective was accomplished by designing, simulating, fabricating, and experimentally investigating the prototype kinematic spindle shown in Fig. 1 [5]. The results presented in this paper demonstrate that the radial error motion is highly repeatable, exhibits low asynchronous error, and that the spindle's synchronous error motion is a predictable function of the roundness profiles in the spindle's rotor.

\section{Background}

An axis of rotation is defined as a line about which rotation occurs [6]. In any real spindle, the rotation is imperfect and the axis of rotation actually displaces and tilts relative to the stator. The motion is described using a set of rigid-body translations and rotations, referred to as error motion. The five rigid-body components of error motion are translations in the $x, y$, and $z$ directions and rotations (or tilting) about the $x$ - and $y$-axes. Except for the master axis method [7], it is difficult to measure these displacements or rotations directly.

Traditional methods for determining error motion use an artifact such as a master cylinder, sphere, or flat mounted to the rotating element of the spindle. The artifact is mechanically adjusted to minimize the once per revolution eccentric- 




Fig. 1. Photograph of a prototype kinematic spindle.

ity or non-squareness with respect to the axis. Runout measurements of the surface of the artifact are then performed as the spindle is rotated. Graphical or digital techniques are used to remove the effects of the remaining artifact eccentricity or non-squareness. For high-precision spindles, the roundness and flatness form errors of the artifact must also be removed. Artifact roundness errors are separated from the spindle radial error motion using the Donaldson [8] reversal technique or multi-probe techniques $[9,10]$, and artifact flatness errors can be separated using the Estler face motion reversal technique $[11,12]$.

Four types of error motion are used to characterize the motion of an axis of rotation relative to the reference axis [6]. Radial error motion is the displacement of an axis of rotation in a direction normal to the Z-reference axis at a specified axial location. Axial error motion is the displacement of the axis of rotation measured coaxial to the Z-reference axis. Tilt error motion is the error motion in an angular direction relative to the $Z$-reference axis. It can be calculated from two radial or axial error motion measurements that are separated by some distance. Face error motion is error motion parallel to the $Z$-reference axis at a specified radial location, and it is a combination of axial error motion and the axial component of tilt motion.

Radial, axial, tilt, and face error motions are often illustrated using polar graphs, where the error motion is plotted against the angular position of the rotor. Total error motion is the complete error motion polar plot as recorded during experimental measurements. It contains both synchronous and asynchronous error motions. Synchronous error motion is the portion of total error motion that occurs at integer multiples of the rotation frequency. Asynchronous error motion is the por- tion of total error motion that occurs at frequencies other than integer multiples of the rotation frequency [11]. The consequences of asynchronous error motion are non-repeatability between consecutive revolutions.

The three most common types of spindles used in highprecision machines and instruments are rolling element, aerostatic, and hydrostatic. Rolling element spindles can exhibit synchronous errors of less than $100 \mathrm{~nm}$, but usually have significantly greater asynchronous error (100-1000 nm) due to the re-circulation of the rolling elements. High quality aerostatic and hydrostatic spindles can exhibit asynchronous errors of less than $5 \mathrm{~nm}$ and synchronous errors of less than $25 \mathrm{~nm}$.

\section{Design of a prototype kinematic spindle}

\subsection{Conceptual design}

A kinematically constrained spindle employs the principles of exact constraint [2] in establishing the position and orientation of a rotating rotor. Therefore, five constraints should be applied to the rotor so that the only free degree of freedom is rotation. One approach to arranging the five constraints is to position one constraint acting axially along the centerline of the rotor and to position the remaining four constraints as two pairs acting radially on the rotor, separated by a finite distance. If the constraints are simple contact points, then a nesting force is necessary to insure that contact always exists.

A conceptual design for establishing such constraints on a rotating rotor is illustrated in Fig. 2. The axial constraint is point contact between an alumina flat surface and a gage ball placed at the end of the rotor. The radial constraints are provided by contact points between the surface of the rotor and four rods that are oriented with their centerlines perpendicular to the rotor's centerline. A nesting force is established in the radial direction by the tension in a compliant drive belt and in the axial direction by permanent magnets that lift the rotor towards the magnetic base. When a torque is transmitted from a servomotor through the compliant belt, the rotor rotates, sliding at the contact points.

\subsection{Detailed design}

The detailed design of a prototype spindle is illustrated in Fig. 3. The $50.8 \mathrm{~mm}$ (2 in.) long shaft was machined from $38.1 \mathrm{~mm}$ (1.5 in.) diameter 440C stainless steel, casehardened to Rc 55-60 (Thomson 60 Case Shaft) with thickness of about $2 \mathrm{~mm}$ (0.08 in.). The shaft was ground and lapped prior to use in the prototype spindle. A distance of $38.1 \mathrm{~mm}$ ( $1.5 \mathrm{in}$.) separates the two contact planes that contain the radial constraints, and the vee-groove angle is nominally $90^{\circ}$.

An O-ring belt (O.D. $3.18 \mathrm{~mm}$ ) wraps around the shaft (rotor), and tension in the belt preloads the rotor against the four radial contact points. Axial preload is established by a 




Fig. 2. Kinematically constraining a spindle rotor with five contact points.

pattern of permanent magnets mounted above the rotor in the top plate. The axial constraint is established by a stainless steel 440C lapped gage ball held in a spherical pocket in the end of the rotor that presses against a flat surface in the endplate. The radial constraints are established by the cylindrical surface of the rotor contacting crossed-cylinders mounted in the vee-groove foundation (spindle stator). Two alternative materials were investigated at the sliding interfaces. First, free-machining 360 brass rods (O.D. $12.7 \mathrm{~mm}$ ) were used. Second, a $0.127 \mathrm{~mm}(0.005 \mathrm{in}$.) thick layer of ultra-high molecular weight polyethylene (UHMW-PE) was bonded to ceramic rods (O.D. $12.7 \mathrm{~mm})$. A $175 \mathrm{~W}(0.23 \mathrm{hp})$ brushless, slotless motor (Aerotech BMS60) and PWM amplifier (Aerotech BA10) rotate the rotor via the O-ring belt. A 3:1 speed reduction is achieved with a $12.7 \mathrm{~mm}$ (0.5 in.) diameter pulley on the motor's shaft. The tension in the $\mathrm{O}$ ring belt is adjustable by sliding the motor mount away from the spindle rotor.

\section{Roundness profiles of this spindle rotor}

In a given cross-section of the rotor, the radial distance between the center of the rotor and points on the rotor surface can be expressed in polar coordinates as the superposition of a circle and an infinite series of periodic functions. Eq. (1) gives the radius of the rotor, $r$, at discrete angular positions, $\theta_{i}$, as a function of the nominal radius, $R$, amplitude of the $k$ th harmonic, $C_{k}$, and phase angle of the $k$ th harmonic, $\Phi_{k}$ $[13,14]$. A specific harmonic term is often referred to as $k$ undulations per revolution (UPR).

$r=R+\sum_{k=1}^{\infty} C_{k} \cos \left(k \theta_{i}-\Phi_{k}\right)$

The first harmonic term ( $k=1 ; 1 \mathrm{UPR})$ is introduced when the centerline of the rotor is not perfectly centered on the axis of rotation while measuring the roundness profile. The second harmonic term $(k=2 ; 2$ UPR) is a combination of the actual 2 UPR harmonic in the rotor and a measurement error that occurs when the rotor centerline is inclined with respect to the axis of rotation (see Appendix A) [15]. Harmonic terms greater than 50 UPR $(k>50)$ are often filtered from the measured profile. For example, the $38.1 \mathrm{~mm}$ diameter rotor used in the prototype spindle would have wavelengths smaller than $2.394 \mathrm{~mm}$ filtered from the measured profile. Therefore, the experimentally measured data collected when measuring roundness profiles actually corresponds to deviations in the radius, $\Delta r$, which are obtained by subtracting the nominal radius, $R$, the first harmonic term, and all harmonic terms greater than 50 UPR from $r$ as shown in Eq. (2). The amplitudes, $C_{k}$, and phase angles, $\Phi_{k}$, can be determined by performing a Fourier transform on the experimentally measured data. 



Fig. 3. Detailed design of a prototype kinematic spindle.

$\Delta r=\sum_{k=2}^{50} C_{k} \cos \left(k \theta_{i}-\Phi_{k}\right)$

Out-of-roundness (OOR) is a common metric used to characterize a particular roundness profile. According to ANSI B89.3.1 [15], the OOR value is defined as the difference between the largest radius and the smallest radius of a measured profile when measured from a common center point.

The roundness profiles from the prototype spindle's rotor were measured in the high and low contact planes, which are identified in Fig. 3. The high and low contact planes correspond to the two cross-sections of the rotor that contact the radial constraints. The measurements were made using methods and an apparatus described by Grejda [16] and software developed by Vigliano [17]. The rotor was rotated using an air-bearing spindle (Professional Instruments 4R) mounted on the worktable of a Moore Universal Measuring Machine. Deviations in the rotor radius were measured using a capacitance displacement gage having a sensitivity of
$1.97 \mathrm{mV} / \mathrm{nm}$ (Lion Precision DMT12) that was attached to the head of the Moore machine. During the measurements, the radial error motion of the spindle was typically less than $7 \mathrm{~nm}$, and the structural error motion was typically less than $18 \mathrm{~nm}$. The noise floor, estimated by combining these two sources using the root sum square (RSS) technique, was about $20 \mathrm{~nm}$. The centers of the roundness profiles were determined using the least squares circle (LSC) method. Deviations in the rotor radius were measured over eight revolutions of the spindle and rotor, and the roundness profile was calculated by averaging the eight individual profiles. The amplitudes and phase angles for the first 50 harmonic terms were calculated using a fast Fourier transformation of the measured data.

The roundness profile measured in the high contact plane of the prototype rotor is illustrated in Fig. 4, and the amplitudes and phases of the first 25 harmonic terms are illustrated in Fig. 5. The roundness profile in the high contact plane exhibited a dominant 2 UPR term with amplitude of $49.7 \mathrm{~nm}$. The OOR in the high contact plane was about $141 \mathrm{~nm}$. 




Fig. 4. Roundness profile measured in the high contact plane of prototype rotor.

The roundness profile measured in the low contact plane of the prototype rotor is illustrated in Fig. 6, and the amplitudes and phases of the first 25 UPR are illustrated in Fig. 7. The amplitudes for the first 20 UPR terms are all significantly larger than those in the high contact plane. Unlike the profile in the high contact plane, this profile exhibited a dominant 4 UPR term with significantly larger amplitude of $105 \mathrm{~nm}$. The OOR value in the low contact plane was about $451 \mathrm{~nm}$, which is about three times larger than the OOR of the high contact plane.

Due to the operations in manufacturing the prototype rotor, the end face of the rotor was not precisely square with the centerline of the rotor. Therefore, the centerline of the rotor was tilted with respect to the axis of rotation in the roundness measurement apparatus. This affected the amplitude and

(a) Amplitude



(b) Phase



Fig. 5. Amplitude and phase for 25 harmonic terms in the roundness profile measured in the high contact plane.



Fig. 6. Roundness profile measured in the low contact plane of prototype rotor.

phase of the measured 2 UPR term in the roundness profile. The measured 2 UPR harmonic, with amplitude, $A$, and phase, $\Phi$, is the superposition of the rotor's actual 2 UPR harmonic (having $A_{1}$ and $\Phi_{1}$ ) and a 2 UPR harmonic resulting from the tilt (having $A_{2}$ and $\Phi_{2}$ ) as shown in Eq. (3) [5]. An explanation of this equation is described in the Appendix A.

$$
\begin{aligned}
& A \cos (2 \theta-\Phi) \\
& =\sqrt{A_{1}^{2}+A_{2}^{2}+2 A_{1} A_{2} \cos \left(\Phi_{1}-\Phi_{2}\right)} \\
& \quad \times \cos \left(2 \theta-\tan ^{-1}\left(\frac{A_{1} \sin \left(\Phi_{1}\right)+A_{2} \sin \left(\Phi_{2}\right)}{A_{1} \cos \left(\Phi_{1}\right)+A_{2} \cos \left(\Phi_{2}\right)}\right)\right)
\end{aligned}
$$

(a) Amplitude



(b) Phase



Fig. 7. Amplitude and phase for 25 harmonic terms in the roundness profile measured in the low contact plane. 


\section{Model and simulation of error motion}

Due to exact constraints, the error motion of a kinematic spindle is deterministic and even predictable a priori knowing the rotor's roundness profiles in the contact planes. As the rotor rotates in contact with its radial constraints, the centers of the rotor in the two contact planes (and hence, the rotor centerline) trace a repeatable trajectory. Assuming that the radial constraints are sufficiently stiff, then the shape of the trajectory depends predominantly on the roundness profiles in the two contact planes. Therefore, the geometry and roundness profiles can be used in a simple model that enables the prediction of the spindle's radial and tilt error motions.

Fig. 8 illustrates the geometry within either the high or low contact plane as the rotor rotates in contact with the radial constraints in the vee-groove. The geometry of the vee-groove is specified by its included angle, $\varphi$. The surface of the rotor contacts the vee-groove at two points labeled $\mathrm{CP}_{1}$ and $\mathrm{CP}_{2}$. A 2D Cartesian coordinate system is defined at the ideal position of the rotor's center, as it would be determined for a perfectly round rotor. Since the rotor profile is imperfect, the center of the rotor translates about the ideal center with its coordinates $\left(\delta_{x}, \delta_{y}\right)$, changing with each increment in the angular position of the rotor, $\gamma_{i}$.

In order for the rotor to contact the vee-groove at $\mathrm{CP}_{1}$ and $\mathrm{CP}_{2}$, the coordinates of the actual center of the rotor, $\left(\delta_{x}, \delta_{y}\right)$, must lie at the intersection of the two lines $L_{1}$ and $L_{2}$. $L_{1}$ and $L_{2}$ are parallel to the $L_{3}$ and $L_{4}$ edges of the vee-groove, so that their slopes are $+m$ and $-m$, respectively. The slope, $m$, depends upon the vee-groove's included angle, $\varphi$, as shown in Eq. (4).

$m=\tan \left(\frac{\pi-\varphi}{2}\right)$

Lines $L_{1}$ and $L_{2}$ pass through the two points $\mathrm{P}_{1}$ and $\mathrm{P}_{2}$, respectively. $\mathrm{P}_{1}$ and $\mathrm{P}_{2}$ lie on lines that radiate from the origin, $\mathrm{O}$, and intersect $\mathrm{CP}_{1}$ and $\mathrm{CP}_{2}$, respectively. The distance between the origin and $\mathrm{P}_{1}$ equals the deviation in the radius of the rotor, $\Delta r_{1}$, measured in the direction of $\mathrm{CP}_{1}$. The devia-



Fig. 8. Geometry for determining location of rotor center in a contact plane. tion, $\Delta r_{1}$, is determined from the roundness profile at angle $\gamma_{i}$ as shown in Eq. (5). Similarly, the distance between $\mathrm{O}$ and $\mathrm{P}_{2}$ equals the deviation in the radius of the rotor in the direction of $\mathrm{CP}_{2}$, which is determined from the roundness profile after shifting $\gamma_{i}$ by $\alpha=(\pi-\varphi)$ as shown in Eq. (6).

$$
\begin{aligned}
& \Delta r_{1}=\sum_{k=2}^{50} C_{k} \cos \left(k \gamma_{i}-\Phi_{k}\right) \\
& \Delta r_{2}=\sum_{k=2}^{50} C_{k} \cos \left(k\left(\gamma_{i}-(\pi-\phi)\right)-\Phi_{k}\right)
\end{aligned}
$$

The Cartesian coordinates of points $\mathrm{P}_{1}$ and $\mathrm{P}_{2}$ are then given for each incremental position of the rotor according to Eqs. (7) and (8), respectively.

$$
\begin{aligned}
& \mathrm{P}_{1}=\left(-\Delta r_{1} \cos \left(\frac{\varphi}{2}\right), \Delta r_{1} \sin \left(\frac{\varphi}{2}\right)\right) \\
& \mathrm{P}_{2}=\left(\Delta r_{2} \cos \left(\frac{\varphi}{2}\right), \Delta r_{2} \sin \left(\frac{\varphi}{2}\right)\right)
\end{aligned}
$$

To determine, the actual center of the rotor, defined by the intersection of lines $L_{1}$ and $L_{2}$, two simultaneous equations are solved. The equations are expressed in matrix form in Eq. (9), and the coefficient, $\beta$, is given in Eq. (10).

$\left[\begin{array}{cc}-m & 1 \\ m & 1\end{array}\right]\left\{\begin{array}{l}x \\ y\end{array}\right\}=\beta\left[\begin{array}{l}\Delta r_{1} \\ \Delta r_{2}\end{array}\right]$

$\beta=m \cos \left(\frac{\varphi}{2}\right)+\sin \left(\frac{\varphi}{2}\right)$

After matrix inversion, Eq. (11) gives the coordinates of the rotor's center $\left(\delta_{x}, \delta_{y}\right)$ in the contact planes for each angular position of the rotor, $\gamma_{i}$.

$\left\{\begin{array}{l}\delta_{x} \\ \delta_{y}\end{array}\right\}=\beta\left[\begin{array}{cc}-\frac{1}{2 m} & \frac{1}{2 m} \\ \frac{1}{2} & \frac{1}{2}\end{array}\right]\left[\begin{array}{l}\Delta r_{1} \\ \Delta r_{2}\end{array}\right]$

The preceding analysis can be performed twice to determine the center of the rotor in both the high contact plane, $\left(\delta_{\mathrm{Hx}}\right.$, $\left.\delta_{\mathrm{Hy}}\right)$, and in the low contact plane, $\left(\delta_{\mathrm{Lx}}, \delta_{\mathrm{Ly}}\right)$. The tilt error of the rotating rotor can then be determined. Eq. (12) gives the rotation about the $X$-axis, $\varepsilon_{x}$, and rotation about the $Y$-axis, $\varepsilon_{y}$, in terms of the locations of the rotor centers in the high and low contact planes and the distance, $l$, separating the two contact planes.

$\left\{\begin{array}{c}\varepsilon_{x} \\ \varepsilon_{y}\end{array}\right\}=\tan ^{-1}\left[\begin{array}{l}\frac{\delta_{\mathrm{Hy}}-\delta_{\mathrm{Ly}}}{l} \\ \frac{\delta_{\mathrm{Hx}}-\delta_{\mathrm{Lx}}}{l}\end{array}\right]$

\subsection{Study 1: trajectory of rotor center and preferred orientation angle}

The preceding approach to determining the trajectory of the rotor's center was used to investigate the shape of the trajectory when the vee-groove's included angle is set to $90^{\circ}$. 




Fig. 9. Trajectory of rotor center for various quantities of lobes in a right-angle vee-groove.

A series of simulations were performed in which the rotor roundness profile was assumed to have only a single harmonic term; this results in a roundness profile exhibiting a dominant quantity of lobes. The first simulation included only the first harmonic term $(k=2)$ and therefore exhibited two lobes; the second simulation included only the second harmonic term $(k=3)$ and therefore exhibited three lobes, etc. The resulting trajectories exhibited one of three possible forms, which are illustrated in Fig. 9. The Cartesian coordinate system corresponds with the coordinate system shown in Fig. 8, so that the $Y$-axis points directly out of the vee-groove. Rotors having $(4 n-2)$ lobes (e.g. 2, 6, 10, etc.) exhibited a trajectory in which the rotor's center oscillated purely along the $X$-axis with a peak displacement of $C_{k} / \cos \left(45^{\circ}\right)$. For rotors with (4n) lobes (e.g. 4, 8, 12, etc.), the trajectory of the rotor's center oscillated in and out of the groove, along the $Y$-axis, with a maximum displacement of $C_{k} / \cos \left(45^{\circ}\right)$. For rotors with $(2 n-1)$ lobes (e.g. $1,3,5$, etc.), the rotor's center traced $(2 n-1)$ circles around the ideal rotor center with a radius equal to the amplitude of the dominant harmonic $\left(C_{k}\right)$.

This pattern of trajectories suggests that kinematic spindles with a $90^{\circ}$ vee-groove may have preferred orientation angles, depending upon the nature of the rotor's roundness profile. A preferred orientation angle is the direction in which the least amount of radial error motion would be measured. If the rotor's roundness profile has $(4 n-2)$ lobes, then the preferred orientation angle would be in the direction of the $Y$-axis, since the trajectory of the rotor's center would mainly be along the $X$-axis. If the roundness profile has dominantly $(4 n)$ lobes, then the preferred orientation angle of the spindle would be in the direction of the $X$-axis since the trajectory of the rotor's center would be mainly along the $Y$-axis. If the spindle's rotor has dominantly $(2 n-1)$ lobes, then the spindle would not exhibit a preferred orientation angle, since the rotor center would tend to trace a circle.

\subsection{Study 2: amplification of rotor out-of-roundness}

In general, the out-of-roundness of the rotor will be amplified in the radial error motion, and the amount of amplification will depend upon both the quantity of dominant lobes and the included angle of the vee-groove. For instance, in Study 1 , where the vee-groove angle was $90^{\circ}$, the gain applied to the amplitude of the harmonic term $\left(C_{k}\right)$ was $1 / \cos \left(45^{\circ}\right)$ for even quantities of lobes or simply 1 for odd numbers of lobes. A second study was conducted to investigate the sensitivity of the gain to both the vee-groove's included angle and various quantities of dominant lobes in the rotor profile. For this study, the gain, $G$, is defined as the ratio of the maximum radial displacement of the rotor's center to the amplitude of the harmonic term in the rotor as given in Eq. (13).

$G=\frac{\max \left(\sqrt{\delta_{x}^{2}+\delta_{y}^{2}}\right)}{C_{k}}$

The gains for a variety of dominantly lobed rotors are plotted in Fig. 10 for vee-groove angles between 45 and $135^{\circ}$ and quantities of lobes ranging from two to eight. This study first reveals that the maximum gain depends upon the quantity of lobes; for instance the maximum gain for a two-lobe rotor is about $G=1.85$ and for a six-lobe rotor is about $G=2.63$. Second, the minimum possible gain for all quantities of lobes is $G=1$, but to achieve this minimum gain, the vee-groove angle must be an appropriate value. For a two-lobe rotor, the best angle is $60^{\circ}$. For a three-lobe rotor, the best angles are 45,90 , and $135^{\circ}$. For a four-lobe rotor, the best angles are 60 and $108^{\circ}$. For a five-lobe rotor, the best angles are 45 , 90 , and $135^{\circ}$. For a six-lobe rotor, the best angles are 77 , 108 , and $128^{\circ}$. Finally, the study also suggests that if there is uncertainty in the quantity of lobes in the rotor roundness profile, then the best vee-groove angle is likely to be $90^{\circ}$ since it offers a compromise minimizing several gains.

\section{Error motion and rotor runout}

\subsection{Predicted trajectory of the rotor's center and surface runout}

The experimentally measured roundness profiles presented in Section 4 were used with the analytical model presented in Section 5 to predict the trajectory of the rotor's center in the high and low contact planes. From this two dimensional trajectory, predictions of the radial error motion in a fixed sensitive direction at different orientation angles and locations can be inferred. The predicted error motions are then combined with the roundness profile to predict the rotor surface runouts. These predictions can be compared directly to experimental measurements.

The roundness profile in the high contact plane (Figs. 4 and 5) contains a dominant two-lobe pattern. As discussed in Study 1 , the trajectory is expected to be predominantly along 




Fig. 10. Amplification of roundness error amplitidue for various quantities of lobes in rotor roundness profile over a range of vee-groove angles.

the $X$-axis. The predicted trajectory is illustrated in Fig. 11, and indeed it tends to traverse about the origin (ideal rotor center) mainly in the $x$-direction. Based on this graph, the radial error motion measured in the high contact plane should be about $\pm 27 \mathrm{~nm}$ in the direction of the $Y$-axis and about $\pm 90 \mathrm{~nm}$ in the direction of the $X$-axis. Hence, the preferred orientation angle would be in the direction of the $Y$-axis.

The roundness profile in the low contact plane (Figs. 6 and 7) had a large four-lobe component, but also had several other significant harmonic components. Based on the discussion in Study 1, the trajectory is likely to translate along the $Y$-axis with some motion in the direction of the $X$-axis. The predicted trajectory in the low contact plane is illustrated in Fig. 12, and indeed it predominantly translates along the $Y$ axis. Unlike the high-contact plane, the trajectory does not exhibit a distinct preferred orientation angle due to the mixture of additional harmonic components. If it could be mea-



Fig. 11. Predicted trajectory of prototype rotor in the high contact plane. sured directly, the radial error motion near the low contact plane would be about 550 and $510 \mathrm{~nm}$ in the $X$ and $Y$ directions, respectively.

The three dimensional trajectory of the rotor's centerline can be also predicted. The results of this prediction visually illustrate the tilt error motion of the axis of rotation as shown in Fig. 13. Each line represents the position of the axis of rotation at discrete positions of the rotor. Due to the high repeatability of the kinematic spindle, the axis of rotation should repeat this error motion with each rotation of the rotor.

\subsection{Measurement of rotor surface runout}

Radial runout of the rotor surface was measured in both the high and low contact planes. This required two enhancements to the prototype spindle, which are shown in Fig. 14:



Fig. 12. Predicted trajectory of prototype rotor in the low contact plane. 




Fig. 13. Predicted trajectory of kinematic spindle's axis-of-rotation.

(1) a rotary encoder (512 counts per revolution) was attached to the spindle rotor and (2) a rigid structure was attached to the spindle housing for mounting capacitance displacement gages (Lion Precision DMT12). A reading from the displacement gage was triggered with each pulse from the rotary encoder, providing 512 displacement readings per revolution. Displacement measurements were recorded during 32 revolutions of the rotor while rotating at a fixed speed of $100 \mathrm{rpm}$.

Displacement gages can be placed in six possible radial locations that are identified in Fig. 15. Three of the gauging locations (points 1, 3, and 5) are located in the high contact plane and three locations (points 2, 4, and 6) are located in the low contact plane. Points 1 and 2 are directly in-line with $\mathrm{CP}_{1}$ and points 3 and 4 are in-line with $\mathrm{CP}_{2}$. Points 5 and 6 are in-line with the $Y$-axis of the coordinate system. The measurements were collected at points 1-6 for the rotor sliding on UHMW-PE and at points 1-3 for the rotor sliding on brass rods.

\subsubsection{Rotor sliding on brass}

The runout measured at point 1 with the rotor sliding on brass is plotted in Fig. 16. The asynchronous and synchronous runouts (cutoff at 50 UPR) in this direction were 25 and $368 \mathrm{~nm}$, respectively.

The amplitude and phase for the first 25 harmonic terms of the runout at point 1 are plotted in Fig. 17. The runout



Fig. 14. Rotary encoder and structure for holding displacement gages during measurement of radial runout.



Fig. 15. Locations for measuring radial runout in high and low contact planes.

exhibited a dominant 2 UPR term and a relatively small 4 UPR term. The amplitudes of the odd UPR terms were negligibly small, so only the even UPR terms of the high contact plane rotor roundness affected the runout at point 1 .

The measured runout at point 2 with the rotor sliding on brass is plotted in Fig. 18. The asynchronous and synchronous error motions at point 2 (cutoff at 50 UPR) were 20 and $889 \mathrm{~nm}$, respectively. The asynchronous runout was a little less than that measured at point 1 , but the synchronous is significantly greater due to the larger out-of-roundness in the low contact plane.

The amplitude and phase for the first 25 harmonic terms of the runout at point 2 are plotted in Fig. 19. The runout exhibited a large 4 UPR term, but also contained significant contributions from the first 10 even harmonic terms. Again, the amplitudes of the odd UPR terms were very small, so only the even harmonic terms of the roundness profile in the low contact plane affected the runout at point 2 .



Fig. 16. Radial runout measured at point 1 with rotor sliding on brass. 
(a) Amplitude



(b) Phase



Fig. 17. Amplitude and phase for first 25 harmonic terms of radial runout measured at point 1 with rotor sliding on brass.

Although the measurement was also conducted at point 3 , plots of the measurements are not presented. These plots were nearly identical to the plots for point 1 with the exception that the data is rotated by $\pi / 2$ radians. A summary of the asynchronous and synchronous runouts measured at each point for consecutive tests is summarized in Table 1. It is evident from this table that the magnitudes of the synchronous and asynchronous runouts exhibited little variation during the consecutive tests.

\subsubsection{Rotor sliding on UHMW-PE bonded to ceramic rods}

The runout measured at point 1 with the rotor sliding on UHMW-PE is plotted in Fig. 20. The asynchronous and



Fig. 18. Radial runout measured at point 2 with rotor sliding on brass. (a) Amplitude



(b) Phase



Fig. 19. Amplitude and phase for first 25 harmonic terms of radial runout measured at point 2 with rotor sliding on brass.

Table 1

Synchronous (syn) and asynchronous (asyn) runout for rotor sliding on brass

\begin{tabular}{|c|c|c|c|c|c|c|}
\hline \multirow[t]{2}{*}{ Trial } & \multicolumn{2}{|c|}{ Point 1} & \multicolumn{2}{|c|}{ Point 2} & \multicolumn{2}{|c|}{ Point 3} \\
\hline & $\begin{array}{l}\text { Syn } \\
(\mathrm{nm})\end{array}$ & $\begin{array}{l}\text { Asyn } \\
(\mathrm{nm})\end{array}$ & $\begin{array}{l}\text { Syn } \\
(\mathrm{nm})\end{array}$ & $\begin{array}{l}\text { Asyn } \\
(\mathrm{nm})\end{array}$ & $\begin{array}{l}\text { Syn } \\
\text { (nm) }\end{array}$ & $\begin{array}{l}\text { Asyn } \\
(\mathrm{nm})\end{array}$ \\
\hline 1 & 404 & 28 & 889 & 25 & 366 & 38 \\
\hline 2 & 379 & 38 & 889 & 20 & 351 & 23 \\
\hline 3 & 394 & 38 & 889 & 25 & 351 & 28 \\
\hline 4 & 368 & 25 & 876 & 43 & - & - \\
\hline
\end{tabular}

synchronous runouts (cutoff at $50 \mathrm{UPR}$ ) in this direction were 41 and $414 \mathrm{~nm}$, respectively. Compared with the measurements at point 1 with the rotor sliding on brass, the asynchronous runout nearly doubled and the synchronous runout



Fig. 20. Radial runout measured at point 1 with rotor sliding on UHMW-PE. 
(a) Amplitude



(b) Phase

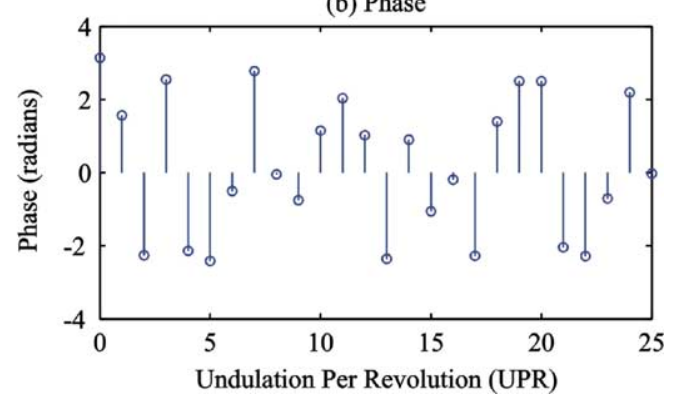

Fig. 21. Amplitude and phase for first 25 harmonic terms of radial runout measured at point 1 with rotor sliding on UHMW-PE.

increased by only $13 \%$. The dramatic increase in the asynchronous value is due to the compliance of the UHMW-PE surface, which allows random fluctuations in belt tension to affect the error motion of the rotor.

The amplitude and phase for the first 25 harmonic terms of the runout at point 1 are plotted in Fig. 21. The radial runout exhibited a dominant 2 UPR term but also contained large 3 UPR and 4 UPR terms. The presence of the odd 3 UPR term suggests that another physical phenomena was significant with the compliant UHMW-PE surface. The amplitudes of the remaining odd UPR terms were very small.

A summary of the asynchronous and synchronous runouts measured at each point for consecutive tests with the rotor sliding on UHMW-PE is listed in Table 2.

\subsection{Comparison of predicted and measured runout}

A comparison of the predicted and measured rotor runout reveals that the error motion is indeed predictable a priori if the roundness profiles of the rotor are known. The comparison presented here is for the runouts measured in the low contact plane with the rotor sliding on brass. A comparison is not conducted with the rotor sliding on UHMW-PE since it exhibited twice as much asynchronous error and since its synchronous error exhibited the irregular 3 UPR harmonic which cannot be explained without violating the rigid contact assumption used in developing the model presented in Section 5. Due to the errors described in Eq. (3) at the end of Section 4 , it is also necessary to remove the 2 UPR harmonic term from the roundness profile and the error motion. The comparison should not be based on the measurements made



Fig. 22. Simulated and measured radial runout (less 2 UPR harmonic) at point 2 with rotor sliding on brass.

in the high contact plane since the roundness and error motion in the high contact plane contained a dominant 2 UPR term. Therefore, the comparison is based on the measurements in the low contact plane at point 2 .

Fig. 22 shows a polar plot of the predicted and measured radial runout at point 2 after the removal of the 2 UPR term, but it is difficult to discern the two curves since they are so close. The maximum and minimum differences are 70 and $-77 \mathrm{~nm}$, respectively. The harmonic components of the difference between the predicted and measured values are plotted in Fig. 23. Here it is evident that the even harmonic terms, and especially the 6 and 8 UPR terms, dominate the discrepancy.

The discrepancy between the predicted and measured runout is partially attributed to errors in the roundness and runout measurements. Several experimental factors also contributed to discrepancy between the predicted and measured

Table 2

Synchronous (syn) and asynchronous (asyn) runout for rotor sliding on UHMW-PE

\begin{tabular}{|c|c|c|c|c|c|c|}
\hline \multirow[t]{2}{*}{ Trial } & \multicolumn{2}{|c|}{ Point 1} & \multicolumn{2}{|c|}{ Point 2} & \multicolumn{2}{|c|}{ Point 3} \\
\hline & $\begin{array}{l}\text { Syn } \\
(\mathrm{nm})\end{array}$ & $\begin{array}{l}\text { Asyn } \\
(\mathrm{nm})\end{array}$ & $\begin{array}{l}\text { Syn } \\
(\mathrm{nm})\end{array}$ & $\begin{array}{l}\text { Asyn } \\
(\mathrm{nm})\end{array}$ & $\begin{array}{l}\text { Syn } \\
(\mathrm{nm})\end{array}$ & $\begin{array}{l}\text { Asyn } \\
(\mathrm{nm})\end{array}$ \\
\hline 1 & 414 & 41 & 775 & 46 & 439 & 53 \\
\hline 2 & 406 & 74 & 782 & 46 & 439 & 51 \\
\hline 3 & 412 & 53 & 780 & 38 & 419 & 48 \\
\hline \multirow[t]{3}{*}{4} & 422 & 53 & - & - & - & - \\
\hline & \multicolumn{2}{|c|}{ Point 4} & \multicolumn{2}{|c|}{ Point 5} & \multicolumn{2}{|c|}{ Point 6} \\
\hline & $\begin{array}{l}\text { Syn } \\
(\mathrm{nm})\end{array}$ & $\begin{array}{l}\text { Asyn } \\
(\mathrm{nm})\end{array}$ & $\begin{array}{l}\text { Syn } \\
(\mathrm{nm})\end{array}$ & $\begin{array}{l}\text { Asyn } \\
(\mathrm{nm})\end{array}$ & $\begin{array}{l}\text { Syn } \\
(\mathrm{nm})\end{array}$ & $\begin{array}{l}\text { Asyn } \\
(\mathrm{nm})\end{array}$ \\
\hline 1 & 805 & 56 & 218 & 41 & 1186 & 43 \\
\hline 2 & 798 & 58 & 221 & 38 & 1184 & 51 \\
\hline 3 & 808 & 69 & 257 & 48 & 1186 & 53 \\
\hline 4 & - & - & - & - & 1194 & 66 \\
\hline
\end{tabular}






Fig. 23. Comparison of amplitudes for 25 harmonic components in simulated and measured radial runout at point 2 with rotor sliding on brass.

values. During the roundness and runout measurements, the typical noise floor was around $20 \mathrm{~nm}$. Additional measurement error is attributed to using a capacitance displacement gage when measuring the roundness of the rotor and its radial runout. First, the gage was calibrated against a flat surface, but it was actually measuring against a cylindrical surface. Also, the round sensing area of diameter $1.70 \mathrm{~mm}$ produced an average measurement over the sensing area, which is larger than the contact area when the rotor touches its radial constraints. Any radial and tilt error motion in the air-bearing spindle used for the roundness measurements introduced errors in the roundness profile.

\section{Conclusions and future work}

A prototype spindle, designed in accordance with the principles of kinematic or exact constraint, was fabricated and experimentally characterized. Since the position and orientation of the spindle rotor is prescribed by four radial constraints and one axial constraint (five contact points), the rotor is free to rotate about its centerline. This paper investigated the radial and tilt error motion of the prototype spindle using an analytical model and experimental measurements.

The synchronous radial error motion of the rotor was shown to predominantly depend upon the roundness profiles in the cross-sectional planes where the four radial constraints contact the rotor. Using a simple analytical model, it was demonstrated that the radial error motion and radial runout of the rotor is even predictable a priori, if the rotor's roundness profiles are known. Two studies using the analytical model reveal the general nature of the synchronous error motion in kinematic spindles. The first study demonstrated three general shapes of trajectories that are traced by the rotor's center when a roundness profile exhibits a dominant quantity of lobes or harmonic terms. If the dominant quantity of lobes is an even number, then the spindle will exhibit a preferred orientation angle in which very little radial error motion will occur. The second study revealed that the maximum radial error for a given rotor roundness profile depends upon the included angle of the vee-groove and the quantity of dominant lobes in the rotor roundness profile, suggesting that the vee-groove angle can be optimally matched with a rotor's roundness profile.

Experimental measurements of the rotor radial runout exhibited very low asynchronous error motion. This attribute is very valuable in applications like metrology, where synchronous errors can be tolerated since they can be compensated or removed, but asynchronous errors can introduce measurement uncertainty. In one direction with the rotor sliding on brass, the asynchronous motion was as low as $25 \mathrm{~nm}$, which approached the noise floor of around $20 \mathrm{~nm}$. This indicates that when the contact points are sufficiently stiff, the radial error motion of kinematic spindles is extremely repeatable; this means that the trajectory of the spindle's axis of rotation is nearly identical with each rotation of the rotor. The asynchronous motion was least for the rotor sliding on brass since the stiffness at the contact points is significantly greater for brass compared to the UHMW-PE surface.

These results suggest that kinematic spindles may have a unique opportunity in instruments and machines that require a low-force and low-speed axis of rotation. For instance, kinematic spindles may provide an inexpensive solution for instruments used in measuring part roundness profiles [18] or within ultra-high vacuums. Current and future work will investigate the effect of other contacting materials (e.g. tungsten carbide sliding on tungsten carbide) and wear at the contact points in order to achieve long-term precision.

\section{Appendix A}

If the axis of a perfectly round shaft (rotor) is tilted with respect to the spindle's axis of rotation while measuring a roundness profile, then the measured profile is elliptical rather than circular when plotted on a polar chart [16]. If the diameter of the shaft, $D$, and angle of tilt, $\psi$, are known, then the shape of the ellipse is given in polar coordinates, $(r, \theta)$, by Eq. (A.1).

$r_{\text {ellipse }}(\theta)=\frac{D / 2}{\sqrt{\cos ^{2}(\psi) \cos ^{2}(\theta)+\sin ^{2}(\theta)}}$

The ellipse can be represented with harmonic terms by expanding Eq. (A.1) into a Fourier cosine series. Eq. (A.2) gives $r_{\text {ellipse }}$ in terms of a nominal radius, $r_{0}$, and the infinite sum of harmonic terms with amplitudes, $a_{k}$, and phases, $\phi_{k}$. The nominal radius, $r_{0}$, is given in Eq. (A.3) and the amplitudes of the harmonic terms are given in Eq. (A.4).

$$
\begin{aligned}
& r_{\text {ellipse }}(\theta)=r_{0}+\sum_{k=1}^{\infty} a_{k} \cos \left(k \theta-\phi_{k}\right) \\
& r_{0}=\frac{1}{\pi} \int_{o}^{\pi} r_{\text {ellipse }}(\theta) \mathrm{d} \theta
\end{aligned}
$$


$a_{k}=\frac{2}{\pi} \int_{0}^{\pi} r_{\text {ellipse }}(\theta) \cos (k \theta) \mathrm{d} \theta$

Evaluation reveals that the odd terms $(k=1,3,5, \ldots)$ equal zero while the even harmonic terms $(k=2,4,6, \ldots)$ are nonzero. Evaluation of the even harmonic terms with Eq. (A.4), when the tilt is small $\left(\psi<1^{\circ}\right)$, reveals that the higher order even terms are negligible compared to the $k=2$ term. It can be shown mathematically that the phase angle for the $k=2$ term, $\phi_{2}$, equals zero, but it is also intuitive since the ellipse is aligned with the direction of tilt. Therefore, the ellipse is well approximated by Eq. (A.5) for small amounts of tilt.

$r_{\text {ellipse }}(\theta)=r_{0}+a_{2} \cos (2 \theta)$

Analytical expressions for the nominal radius, $r_{0}$, and the amplitude of the second harmonic term, $a_{2}$, are given by Eqs. (A.6) and (A.7) in terms of complete elliptic integrals of the first kind, $K(e)$, and second kind, $E(e)$, which are based on the modulus, $e$, given in Eq. (A.8).

$r_{0}=\frac{D}{\pi} K(e)$

$a_{2}=\frac{2 D}{\pi e^{2}}\left[\left(-e^{2}+2\right) K(e)-2 E(e)\right]$

$e=\sqrt{1-\cos ^{2}(\psi)}$

For a particular rotor diameter, $D$, the amplitude, $a_{2}$, increases with the amount of tilt, $\psi$; this causes the ellipse to elongate so that the distance between the ellipse foci increases. Fig. 24 shows the dependence of $a_{2}$ on $\psi$ for a shaft having a diameter equal to the rotor of the prototype kinematic spindle ( $D=38.1 \mathrm{~mm}$ ). The $k=2$ harmonic given in Eq. (A.5) is effectively an erroneous 2 UPR term that is present within the measured profile, and Fig. 24 demonstrates that the term may not be negligible for high-precision shafts or significant tilt.

The effect of the erroneous 2 UPR term on the measured 2 UPR term is revealed by superposition. The superposition of two harmonic terms with the same frequency produces a new harmonic with the same frequency but a different amplitude and phase. If the rotor of the kinematic spindle was



Fig. 24. Ratio of erroneous 2 UPR harmonic term to shaft diameter as function of tilt angle. tilted by angle, $\psi$, at a phase angle, $\phi$, with respect to the angular reference marker on the instrument's spindle, then the erroneous 2 UPR term is given by Eq. (A.9), which is rewritten in terms of the amplitude, $A_{2}$, and phase angle, $\Phi_{2}$. This term would be superposed with the rotor's actual 2 UPR term with amplitude, $A_{1}$, and phase angle, $\Phi_{1}$, given in Eq. (A.10). The phase angles, $\Phi_{1}$ and $\Phi_{2}$, are measured with respect to the reference marker on the instrument's spindle.

$r_{\mathrm{err}}(\theta)=a_{2} \cos (2(\theta-\phi))=A_{2} \cos \left(2 \theta-\Phi_{2}\right)$

$r_{\text {act }}(\theta)=A_{1} \cos \left(2 \theta-\Phi_{1}\right)$

The superposition of the erroneous and actual 2 UPR terms produces a measured 2 UPR harmonic term, given in Eq. (A.11), with amplitude, $A$, and phase angle, $\Phi$, that are given in Eqs. (A.12) and (A.13), respectively.

$r_{\text {meas }}(\theta)=A \cos (2 \theta-\Phi)$

$A=\sqrt{A_{1}^{2}+A_{2}^{2}+2 A_{1} A_{2} \cos \left(\Phi_{1}-\Phi_{2}\right)}$

$\Phi=\tan ^{-1}\left(\frac{A_{1} \sin \left(\Phi_{1}\right)+A_{2} \sin \left(\Phi_{2}\right)}{A_{1} \cos \left(\Phi_{1}\right)+A_{2} \cos \left(\Phi_{2}\right)}\right)$

From Eq. (A.12), it is evident that the amplitude of the measured 2 UPR harmonic, $A$, can be greater or less than the amplitude of the actual 2 UPR term, $A_{1}$. Furthermore, the phase of the measured 2 UPR term, $\Phi$, can be shifted with respect to other measured harmonic terms in the roundness profile.

\section{References}

[1] Slocum AH. Precision machine design. Dearborn, MI: Society of Manufacturing Engineers; 1992.

[2] Blanding DL. Exact constraint: machine design using kinematics principles. New York: ASME Press; 1999.

[3] Lindsey K, Smith ST, Robbie CJ. Sub-nanometer surface texture and profile measurement with 'Nanosurf 2'. Ann CIRP 1988;37(1):519-22.

[4] Evans CR, Polvani RS, Scire FE, Ruff AW, Hu XZ, Teague EC. Way systems for the molecular measuring machine. In: Proceedings of the Fifth International Precision Engineering Seminar, Monteyrey, CA, 18-22 September 1989.

[5] Hii KF. Kinematically constrained spindle. Master's thesis. University of Kentucky, Lexington, Kentucky, 2002.

[6] ANSI/ASME B89.3.4M. Axes of rotation-methods for specifying and testing. New York: American Society of Mechanical Engineers; 1985.

[7] Marsh ER, Grejda RD. Experiences with the master axis method for measuring spindle error motions. Prec Eng 2000;24(1):50-7.

[8] Donaldson R. A simple method for separating spindle error from test ball roundness error. Ann CIRP 1972;21(2):125-6.

[9] Shinno H, Mitsui K, Tatsue Y, Tanaka N, Tabata T, Omino T. New method for evaluating error motion of ultraprecision spindle. Ann CIRP 1987;36(1):381-4.

[10] Whitehouse DJ. Handbook of surface metrology. Bristol, PA: Institute of Physics Publication; 1994.

[11] Draft of ANSI/ASME B89.3.4M. Axes of rotation-methods for specifying and testing. New York: American Society of Mechanical Engineers; 2002.

[12] Salsbury JG. Implementation of the estler face motion reversal technique. Prec Eng 2003;27(2):189-94.

[13] Damir MNH. Approximate harmonic models for roundness profiles. Wear 1979;57:217-25. 
[14] Cho N, Tu J. Roundness modeling of machined parts for tolerance analysis. Prec Eng 2001;25:35-47.

[15] ASME/ANSI B89.3.1. Measurement of out-of-roundness. New York: American Society of Mechanical Engineers; 1972.

[16] Grejda RD. Use and calibration of ultra precision axes of rotation with nanometer level of metrology. Ph.D. thesis. Penn State University, State College, PA, 2002.
[17] Vigliano CV. Computer control for precision bearing analysis. Masters thesis. Penn State University, State College, PA, May 2001.

[18] Wolsing E, Hii KF, Vallance RR. Kinematic spindles for portable roundness instruments. In: Proceedings of the 2002 Annual Meeting of the American Society for Precision Engineering, St. Louis, MO, October 2002. 